\title{
Real-world data on Rett syndrome: Q\&A with Jana von Hehn
}

\section{BY PETER HESS}

8 FEBRUARY 2022

Rett syndrome affects about 1 in 10,000 female babies, for whom there are no approved medications. (The condition rarely affects boys.) Clinical trials of drugs to treat the condition's core features - which include motor, eating and breathing problems, and autism - are underway, but it can be challenging to recruit large groups of participants with the rare condition and to collect reliable data on their traits.

Jana von Hehn, senior director of research and clinical strategy for the Rett Syndrome Research Trust, aims to ease those challenges with the Rett Syndrome Global Registry, a centralized database containing information from caregivers and medical records. It has been set up to incorporate additional data from wearable sensors and other sources, as those data become available. The registry is slated to launch this year, and researchers and drug companies could use it in ongoing clinical trials and to find participants for future studies, von Hehn says.

Spectrum spoke to her about the motivation behind the registry, as well as the trust's plans for its future.

\section{Spectrum: Why did you create this registry?}

Jana von Hehn: For years, we've been trying to figure out how we can best support pharmaceutical companies that are considering developing therapeutics for Rett syndrome. We realized there are a lot of untapped data sources that we could synthesize to help pharmaceutical companies inform clinical trials and enroll the appropriate participants. In our effort to do this as efficiently as possible, we came up with the Rett Syndrome Global Registry, which combines available untapped data and makes them research-ready.

\section{S: What kind of data will the registry contain?}


JvH: One is medical records that sit essentially unused at doctors' offices everywhere. The other is all the day-to-day data that parents have from caring for their loved ones. We'll have a website and an app in which parents can enter information about their experience living with and caring for someone who has Rett syndrome. This includes their care strategies, important medical events, how they feel about clinical trials, what their family life is like, and their child's traits and how they change. This information is meant to add context to what is reported in medical records.

For the medical records, we're working with Ciitizen, a data aggregation company with a proprietary artificial intelligence system that can consolidate and digitize medical records. Our goal is to turn all the aggregate data into a clinical-trial-grade registry.

\section{S: What exactly does 'clinical-trial-grade' mean?}

JvH: My team and I come from the pharmaceutical industry, so we know the type of data and data quality that pharma companies need to run a clinical trial or submit their data to the U.S. Food and Drug Administration (FDA). A lot of the blockades that registries typically have is that they don't collect data with the same rigor that clinical trials do. So we partnered with a global clinical trial database provider, DSG, Inc, whose databases have the highest security standards and meet FDA and other regulatory agency requirements for electronic data that would be typical for a clinical trial.

\section{S: Now that the infrastructure has been built, what are the trust's goals for the registry?}

JvH: The short-term goal for the registry is just getting people enrolled. The value of the registry and the data it contains will only grow if we have a lot of data. Because it's a fully remote system, we can enroll participants from all over the world and really get the numbers up for this rare disease.

Our longer-term goal is to support pharma companies by making our data accessible to help support clinical development. This will include data summaries that researchers and pharma companies can access easily.

\section{S: How will the registry help Rett syndrome drug trials?}

JvH: Any study could benefit from a deeper understanding of the Rett syndrome population. How many people have had genetic testing? How many participants meet clinical diagnostic criteria, and how many would be atypical? What are the top trait concerns? What should researchers be measuring in their clinical trials? There's a lot of information that could help them understand the feasibility of the trial design they're planning and help them design the study so they're assessing for the right endpoint.

People who are interested in participating could receive caregiver-based questionnaires, or even biosensors, and collect the data at home. This type of research can help develop new ways to 


\section{Spectrum | Autism Research News}

https://www.spectrumnews.org

measure changes in traits that could make clinical trials better at detecting meaningful improvements. Then we can link the data collected that way to the registry database, where they can be analyzed any which way for whatever symptom we're assessing - sleep mobility, heart rate, breathing rate, all these different things - and generate a rich dataset to mine for trends.

One of our goals is to eventually have the data or the participants in our system serve as a concurrent control arm for clinical trials, helping to get therapeutics to market faster.

\section{S: How would that work?}

JvH: We're planning to get longitudinal data from our registrants to understand normal trait variability over time, which will enable us to generate data on the same timescales as clinical trials are conducted. And if there are specific assessments being done in a clinical trial, we could have those added into the registry. Then we would recruit our participants who meet the trial's inclusion criteria and have them participate in the same activities as those in the trial.

Essentially, we would be generating data concurrently with the trial, using a participant pool that may be larger and more representative of an untreated population than what would be feasible to enroll in the trial.

\section{S: So the idea is that registrants will contribute data to a trial, even though they are not formally participating in a traditional sense at the trial site?}

JvH: Right, researchers will be able to identify potential participants from a broad, global population, based on select criteria already available, such as mutation, trait manifestation, age, etc., so the chances of quickly finding people who meet the entrance criteria for the trial are going to be high. Then, if there's anything specific, such as blood draws or other types of clinic-based assessments, we can build those in. They are not as easy to do as something like a parent questionnaire, but it's not impossible.

\section{S: What have been the biggest challenges to getting the registry up and running?}

JvH: It has been difficult trying to be as comprehensive as possible, coming up with every trait a participant might have and every way the parents might manage it or want to track it.

We also want parents to have a unique experience and enjoy being part of the registry, so we've spent a good amount of time with our parent committee trying to figure out what's going to make people want to join the registry. For example, we have trait trackers and logs available in an app for this registry. They can then use that information over time to work with their clinicians to improve their loved one's day-to-day care. And, say they're on vacation or away from home and they have to go to the emergency room, they can pull up information that might be informative for a physician, right from their phone. We're also enabling them to learn about how other families manage traits 


\section{Spectrum | Autism Research News}

https://www.spectrumnews.org

and connect with other families through the registry.

\section{S: Once the registry is up and running, how will researchers and drug companies be able to use it?}

JvH: We would like to start with multiple data points on 500 to 1,000 people, which we think will make the data useful and representative. Once we hit that threshold, we're going to have what's called a global dashboard for the registry. Families can also access it, but it will be a special dashboard for researchers and pharma companies, where they can see all the aggregate data in the system, with the ability to filter down into any particular interest. We want to have summaries that can inform trial design, feasibility, endpoint selection and quality of life, all readily accessible for researchers and pharma companies.

They can also formally request data if they need more details than the global dashboard provides, and we can provide them with anonymized datasets to help make decisions, conduct their own research, write papers or whatever it is they'd like to do to try to move the field forward.

Cite this article: https://doi.org/10.53053/WGWC5332 